\title{
Middle lobe torsion after right upper lobectomy: A report of video-assisted thoracoscopic management
}

\author{
Charles C. Sticco, D0, Shahriyour Andaz, MD, FACS, and Stewart Fox, MD, FACS, Oceanside, NY
}

$\mathrm{T}$ he incidence of postlobectomy lobar torsion is rare. The literature states that lobe torsion occurs in $0.089 \%$ to $0.3 \%$ of patients after lung resection. ${ }^{1}$ Traditionally, treatment includes re-exploratory thoracotomy with affected lobe resection. ${ }^{2}$ We present a case of right middle lobe torsion with surgical treatment through a video-assisted thoracoscopic (VATS) approach after thoracotomy and right upper lobectomy.

\section{Clinical Summary}

A 59-year-old male patient had chest pain and cough for 10 days. A chest radiograph revealed a small round nodular density in the right upper lobe of the lung. An outpatient computed tomographic (CT) scan confirmed the presence of a $1.7-\mathrm{cm}$ speculated tumor with no significant mediastinal lymphadenopathy. Medical history was significant for Crohn disease, hypertension, and a 45 packyear history of smoking. Home medications included valsartan (Diovan), mesalamine (Asacol), and methotrexate.

The patient initially underwent a right VATS with wedge resection followed by a right thoracotomy and formal right upper lobe lobectomy. Pathologic examination revealed moderately differentiated adenocarcinoma without lymph node involvement staged as a T1 N0 tumor.

On postoperative day 2 , the patient became tachypneic, tachycardic, and reported having a cough with dark red sputum. Oxygen saturation was $90 \%$ on $50 \%$ supplemental oxygen.

Arterial blood gases revealed a carbon dioxide tension of 36 $\mathrm{mm} \mathrm{Hg}$ and an oxygen tension of $54 \mathrm{~mm} \mathrm{Hg}$. A chest radiogram demonstrated a large opacity occupying the medial right hemithorax most likely representing a collapsed right middle lobe (Figure 1).

An examination with a flexible bronchoscope was performed at this time. Aspiration of mucus plugs revealed possible kinking and occlusion of the right distal bronchus. After the procedure, the patient's hemodynamic and clinical status had not improved and the decision to perform a second operation was made at this time. The chest was re-explored thoracoscopically, and the diagnosis of right middle lobe torsion was confirmed. A venous congested right

\footnotetext{
From the Department of Thoracic Surgery, Soouth Nassau Communities Hospital, Oceanside, NY.

Received for publication May 16, 2007; accepted for publication May 30, 2007.

Address for reprints: Charles C. Sticco, DO, General Surgery Resident, South Nassau Communities Hospital, One Healthy Way, Oceanside, NY 11572 (E-mail: csticco@ numc.edu).

J Thorac Cardiovasc Surg 2007;134:1090-1

$0022-5223 / \$ 32.00$

Copyright $\odot 2007$ by The American Association for Thoracic Surgery doi:10.1016/j.jtcvs.2007.05.046
}

middle lobe was visualized and appeared to be turned $360^{\circ}$ around the bronchovascular pedicle (Figure 2). The affected lobe was reduced. The congestion failed to resolve and a right middle lobectomy via VATS was performed.

The postoperative course was uneventful. The patient was discharged on postoperative day 13 from the initial operation. Histopathologic examination of the right middle lobe revealed diffuse gangrenous changes and vascular dilatation filled with blood clot.

\section{Discussion}

The true incidence of lobar torsion has been difficult to determine. Published reports have documented torsion developing in $0.089 \%$ to $0.3 \%$ of patients who have had lobectomy. ${ }^{1}$ The exact pathophysiology of lobar torsion remains controversial. Early articles have shown that bronchial occlusion produces ischemia of lobar bronchi, whereas other studies describe venous outflow obstruction and blood flow interruption causing parenchymal congestion and ischemia seen in lobar torsion. ${ }^{1}$

The clinical presentation of lobar torsion is varied. Cable and associates ${ }^{1}$ described how signs and symptoms may present dramatically with fever, tachycardia, and loss of breath sounds over the affected lung field. Other patients may exhibit these characteristics to a lesser degree. Respiratory distress usually coincides with radiographs showing opacification of the affected lung. It has been suggested that suturing the middle lobe to the lower lobe after a right upper lobectomy may prevent lobar torsion. ${ }^{1}$ Although this was preformed in our case, middle lobe torsion still occurred. Previous case reports have described this presentation immediately after surgery as well as several days postoperatively. ${ }^{1-3}$ This report illustrates how a high degree of awareness and prompt treatment ensures a satisfactory outcome through a minimally invasive procedure.

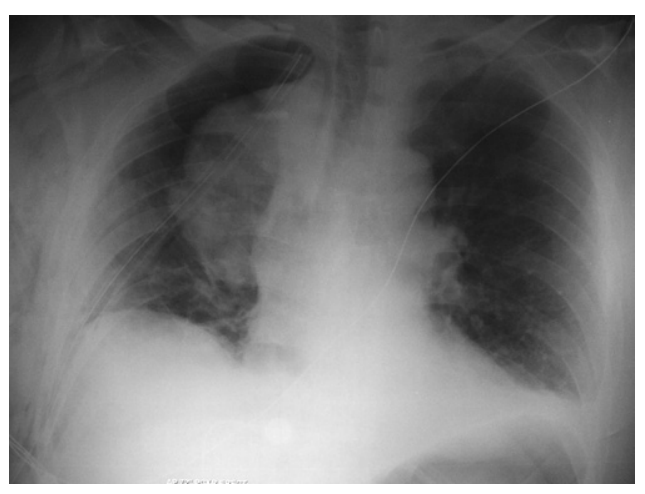

Figure 1. Chest radiogram demonstrating large opacity occupying the medial right hemithorax most likely representing a collapsed right middle lobe on postoperative day 2 . 


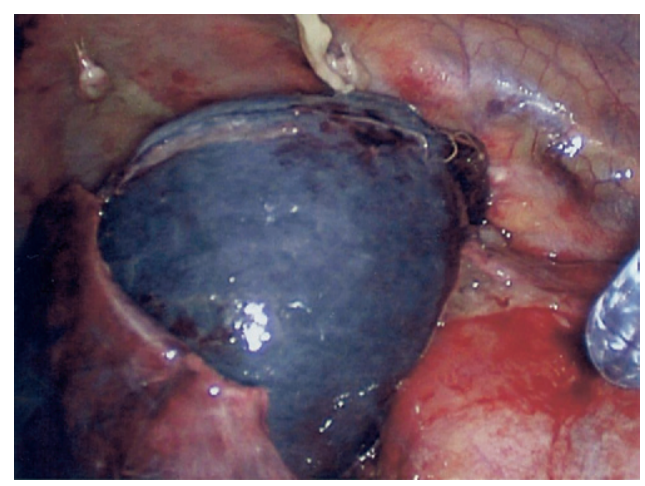

Figure 2. Thoracoscopic visualization of venous congested right middle lobe secondary to torsion.

Current treatment of lung torsion is thoracotomy with lung resection of the nonviable lobe. ${ }^{2}$ The indications for thoracoscopic lobectomy have been expanding with increasing surgeon experience. VATS lobectomy may offer a second option with possible benefits over the open technique. McKenna, Houck, and Fuller ${ }^{4}$ have noted a decrease in blood loss, less postoperative pain, and better pulmonary function after surgery in patients undergoing VATS lobectomy. As minimally invasive thoracic surgery becomes more readily available, the literature is mounting that shows reduced rates of complications and better survival. ${ }^{4}$

Our patient's uneventful course postoperatively may indicate a suitable alternative to open thoractomy for treating lobar torsion. This case occurred after open thoracotomy; however, as VATS lobectomy becomes more prevalent for non-small cell lung cancer and other indications, the occurrence of lobar torsion after VATS should be investigated. Additional reporting is necessary to determine if this less invasive technique results in quicker recovery and in a better overall outcome.

\section{References}

1. Cable D, Deschamps C, Allen M, Miller D, Nichols F, Trastek V, et al. Lobar torsion after pulmonary resecton: presentation and outcome. J Thorac Cardiovasc Surg. 2001;122:1091-3.

2. Demir A, Akin H, Olemen A, Melek H, Dincer S. Lobar torsion after pulmonary resection: report of two cases. Ann Thorac Cardiovasc Surg. 2006;12:63-5.

3. Kanaan S, Boswell W, Hagen J. Clinical and radiographic signs lead to early detection of lobar torsion and subsequent successful intervention. J Thorac Cardiovasc Surg. 2006;132:720-1.

4. McKenna R, Houck W, Fuller C. Video-assisted thoracic surgery lobectomy: experience with 1,100 cases. Ann Thorac Surg. 2006;81: 421-5. 